\title{
Identification of the Kappa-Opioid Receptor as a Therapeutic Target for Oligodendrocyte Remyelination
}

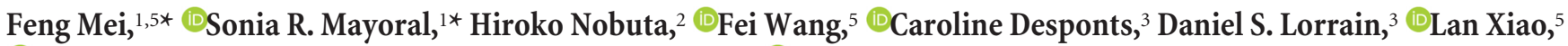 \\ ๑Ari J. Green, ${ }^{1,4}$ David Rowitch, ${ }^{2}$ Jennifer Whistler, ${ }^{1,6}$ and $\odot$ Jonah R. Chan ${ }^{1,6}$ \\ ${ }^{1}$ Department of Neurology, University of California, San Francisco, California 94158, ${ }^{2}$ Department of Pediatrics and Neurosurgery, Eli and Edythe Broad \\ Institute for Stem Cell Research and Regeneration Medicine and Howard Hughes Medical Institute, University of California, San Francisco, California \\ 94143, ${ }^{3}$ Inception Sciences, San Diego, California 92121, ${ }^{4}$ Department of Ophthalmology, University of California, San Francisco, California 94158, \\ ${ }_{5}^{5}$ Department of Histology and Embryology, Chongqing Key Laboratory of Neurobiology, Third Military Medical University, Chongqing, 400038, China, and \\ ${ }^{6}$ Program in Neuroscience, University of California, San Francisco, California 94158
}

Remyelinating therapies seek to promote restoration of function and normal cellular architecture following demyelination in diseases, such as multiple sclerosis (MS). Functional screening for small molecules or novel targets for remyelination is a major hurdle to the identification and development of rational therapeutics for MS. Recent findings and technical advances provide us with a unique opportunity to provide insight into the cell autonomous mechanisms for remyelination and address this unmet need. Upon screening a G-protein-coupled receptor small-molecule library, we report the identification of a cluster of $\kappa$-opioid receptor (KOR) agonists that significantly promotes oligodendrocyte differentiation and myelination. KOR agonists were validated in purified rat oligodendroglial cultures, and the $( \pm) \mathrm{U}-50488$ compound proved to be most effective for differentiation. $( \pm) \mathrm{U}-50488$ treatment significantly enhances differentiation and myelination in purified oligodendroglial cocultures and greatly accelerates the kinetics of remyelination in vivo after focal demyelination with lysolecithin. The effect of $( \pm) \mathrm{U}-50488$ is attenuated by KOR antagonists and completely abolished in KOR-null oligodendroglia. Conditional deletion of KOR in murine oligodendrocyte precursor cells (OPCs) greatly inhibits remyelination after focal demyelination lacking any response to $( \pm) \mathrm{U}-50488$ treatment. To determine whether agonism of KOR represents a feasible therapeutic approach, human induced pluripotent stem cell-derived OPCs were treated with $( \pm) \mathrm{U}-50488$. Consistent with findings, differentiation of human OPCs into mature oligodendrocytes was significantly enhanced. Together, KOR is a therapeutic target to consider for future remyelination therapy.

Key words: kappa-opioid receptor; oligodendrocytes; remyelination

\section{Significance Statement}

Remyelination represents a promising strategy to achieve functional recovery in demyelinating diseases, like MS. Thus, identification of potent compounds and targets that promote remyelination represents a critically unmet need. This study reports a cluster of compounds that are highly effective in enhancing remyelination and identifies $\kappa$-opioid receptor (KOR) as a positive regulator for oligodendroglial differentiation, implicating KOR agonism as a potential strategy to accelerate remyelination.

\section{Introduction}

Myelin is a unique component of the nervous system that not only maximizes the efficiency and velocity of neuronal action

Received May 6, 2016; revised June 9, 2016; accepted June 12, 2016.

Author contributions: F.M., S.R.M., H.N., A.J.G., and J.R.C. designed research; F.M., S.R.M., H.N., F.W., and J.R.C. performed research; F.M., S.R.M., H.N., C.D., D.S.L., L.X., A.J.G., D.R., J.W., and J.R.C. contributed unpublished reagents/analytic tools; F.M., S.R.M., H.N., and J.R.C. analyzed data; F.M., S.R.M., H.N., and J.R.C. wrote the paper.

This work was supported by the US National Multiple Sclerosis Society (RG5203A4), NIH/NINDS (R01NS062796), the Rachleff Endowment to J.R.C., the Overseas Youth Program Grant of the Third Military Medical University and the National Natural Science Foundation of China (NSFC81270017) to F.M., the NIH/NIGMS (K12GM081266) IRACDA Postdoctoral Fellowship to S.R.M., and the Joint Research Fund for Overseas Chinese Young Scholars (NSCF 31228011) Chongqing Scientific and Technical Innovation Foundation of China (CSTCKJCXLJRCO7) to L.X. We thank potentials, but also plays fundamental roles in modulating information flow within neural circuits, as well as providing axonal support (Lappe-Siefke et al., 2003; Fünfschilling et al., 2012; Y.

the members of the Chan laboratory and the MS Research Group at UCSF for support, advice, and insightful discussions.

The authors declare no competing financial interests.

*F.M. and S.R.M. contributed equally to this work.

Correspondence should be addressed to Dr. Jonah R. Chan, Department of Neurology and Program in Neuroscience, University of California San Francisco, Sandler Neurosciences Center, 675 Nelson Rising Lane, Room 214C, Box 3206, San Francisco, CA 94158. E-mail: jonah.chan@ucsf.edu.

D. Rowitch's present address: Department of Pediatrics, Wellcome Trust-MRC Stem Cell Institute, University of Cambridge, $\mathrm{CB} 2 \mathrm{OAH}, \mathrm{UK}$.

DOI:10.1523/JNEUROSCI.1493-16.2016

Copyright $\odot 2016$ the authors $\quad 0270-6474 / 16 / 367925-11 \$ 15.00 / 0$ 
a

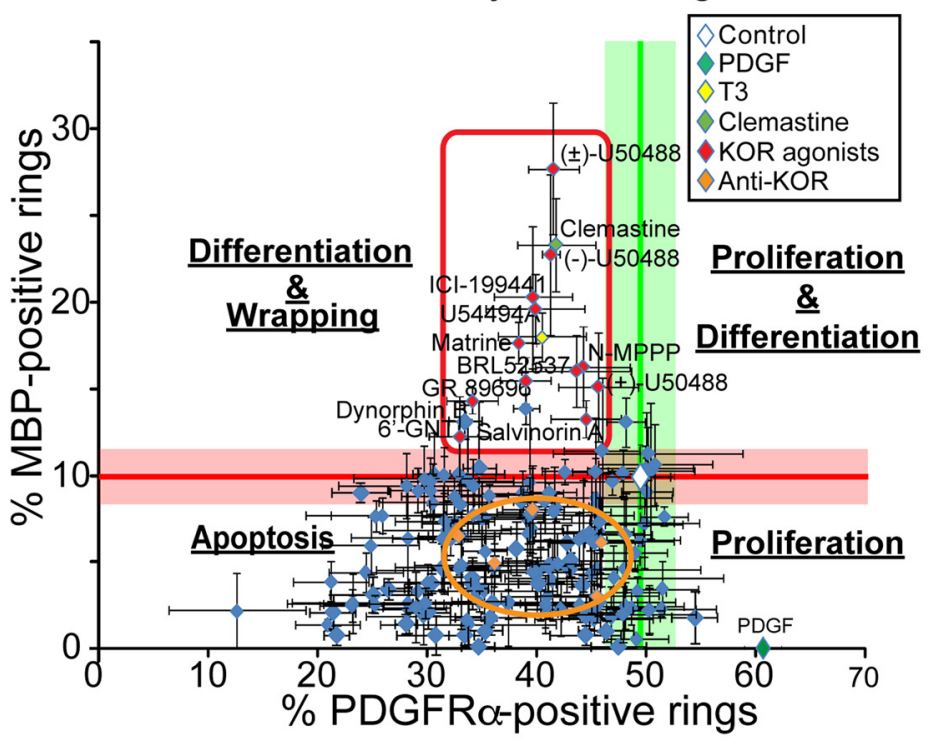

b Control

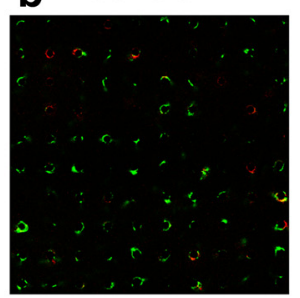

KOR antagonists

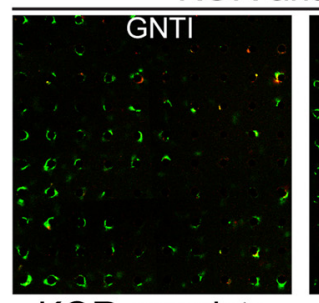

KOR agonists
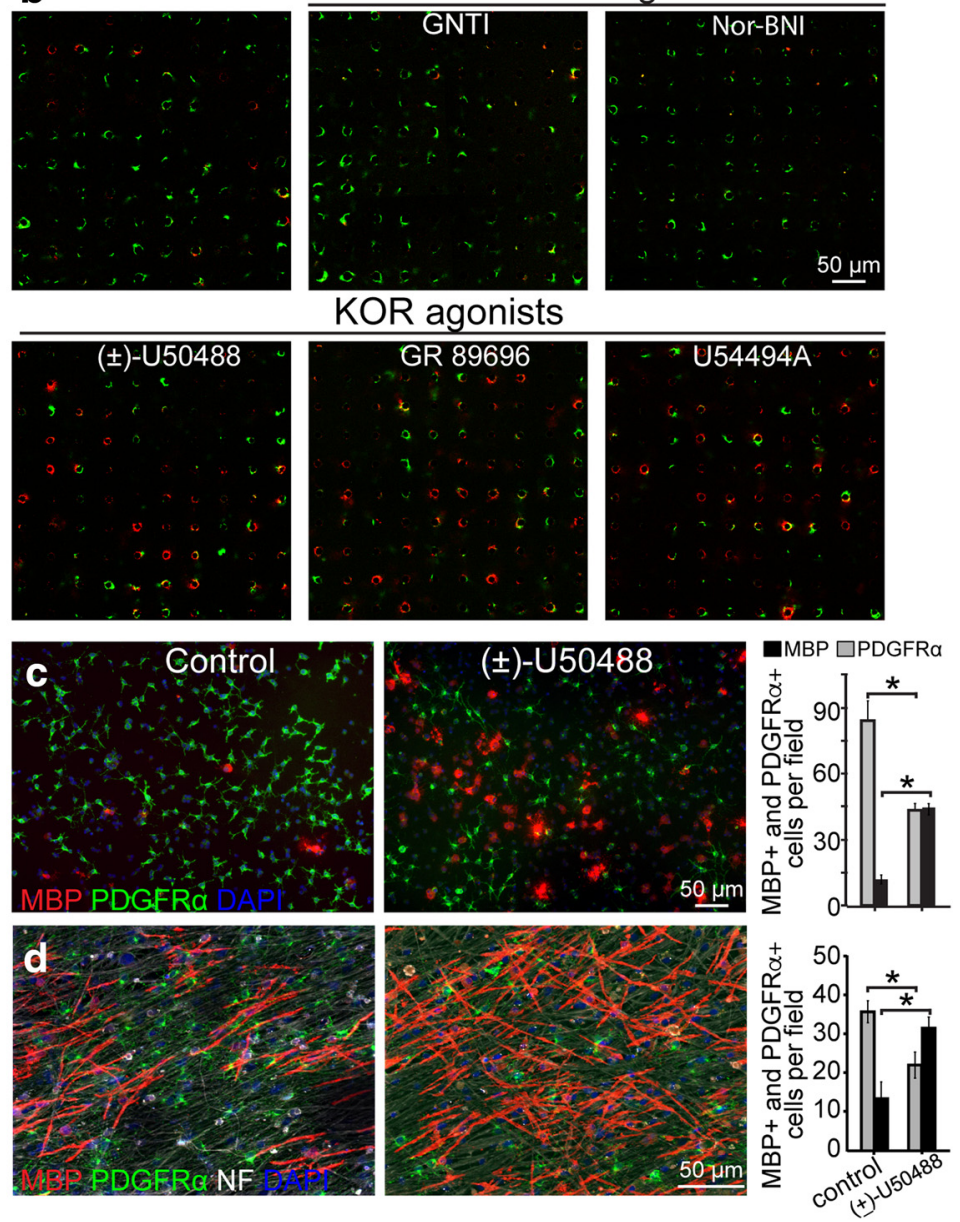

Figure 1. Screening a GPCR library to identify a cluster of KOR agonists and validation of ( \pm )-U50488 with purified OPCS cultured alone or cocultured with DRG neurons. $\boldsymbol{a}$, Two-hundred-fifty compounds were plotted based on the percentage of MBP-positive and PDGFR $\alpha$-positive rings per 100-micropillar-containing field. Compounds were categorized into four quadrants representing increased proliferation, apoptosis, differentiation and wrapping, and the combination of both proliferation and differentiation, from control levels. The control (white diamond) is represented at the intersection of the green and red lines and indicates the average measurement of MBP-positive and PDGFR $\alpha$-positive rings obtained with vehicle treatment. SEM values are indicated by the width of the red and green lines for the vehicle control. $\boldsymbol{b}$, Representative 100 micropillar fields immunostained for MBP (red) and PDGFR $\alpha$ (green) for control arrays, KOR antagonists (GNTI and nor-BNI), and KOR agonists [( \pm )-U50488, GR89696,
Lee et al., 2012; Morrison et al., 2013; Saab et al., 2013). After demyelinating insult, remyelination can occur spontaneously, albeit inefficiently, as oligodendrocyte precursor cells (OPCs) are widely distributed throughout the adult CNS and maintain their potential to differentiate into mature oligodendrocytes (OLs) and myelinate axons (Zhang et al., 1999; Nunes et al., 2003). Remyelination by adult OPCs does not appear to be hampered solely by issues of access or recruitment (Keirstead et al., 1998; Scolding et al., 1998; Wolswijk, 1998; Chang et al., 2000; Woodruff et al., 2004). This underscores that a failure of OPC differentiation and myelination, rather than recruitment and/or migration of OPCs, is the principal barrier impeding remyelination in demyelinating conditions. Functional screening for small molecules or biologicals that promote remyelination represents a major hurdle to the identification and development of rational therapeutics for multiple sclerosis (MS) and recently has received much attention (Deshmukh et al., 2013; Najm et al., 2015). Identifying novel therapeutics and promising targets will impart valuable insight in enhancing stability and repair of myelin in diseases, such as MS, and ultimately promote functional recovery.

In this study we identified a cluster of compounds that are highly potent in promoting remyelination by oligodendrocytes, upon screening a G-protein-coupled receptor (GPCR) small-molecule library. Our results indicate the $\kappa$-opioid receptor (KOR) as a positive regulator for oligodendroglial differentiation and myelination, which may be a potential therapeutic target for remyelination.

\section{Materials and Methods}

Transgenic mice. All mice examined in this study were handled in accordance with the approval of the University of California San Francisco Administrative Panel on Laboratory Animal Care. KOR knock-out mice were purchased from The Jackson Laboratory (Catalog \#007558). The KOR floxed and Olig2-Cre mice were described previously (Chefer et al., 2013;

and U54494]. c, Representative images and quantification of purified rat $\mathrm{OPC}$ cultures treated with vehicle (control) or ( \pm )U50488 for $48 \mathrm{~h}$ and immunostained for MBP (red) and PDGFR $\alpha$ (green). $\boldsymbol{d}$, Representative images and quantification of OPC-DRG neuron cocultures treated with vehicle (control) or ( \pm )-U50488 for $5 \mathrm{~d}$ and immunostained for MBP (red), PDGFR $\alpha$ (green), and neurofilament (white). Cell nuclei are identified by DAPI (blue). Error bars represent mean \pm SEM. ${ }^{*} p<0.05$; significance based on Student's $t$ test. $N=3$ for all experiments. 
CTL
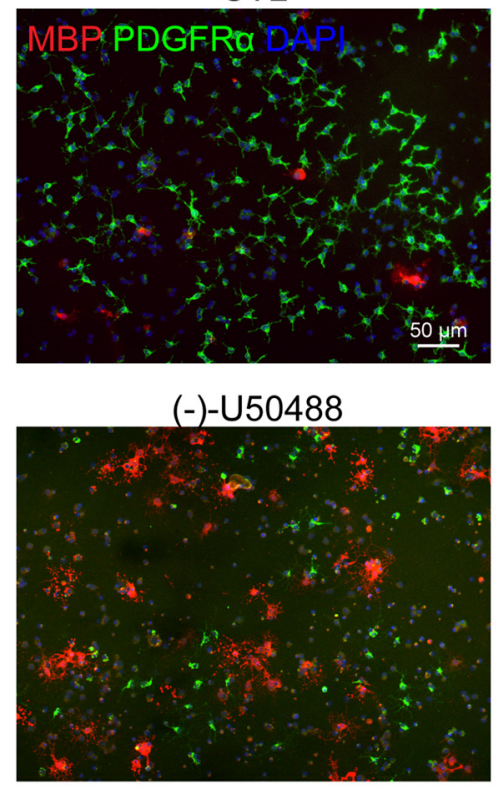

GR 89696

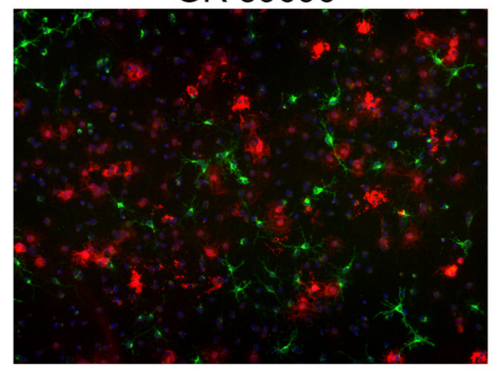

(+)-U50488
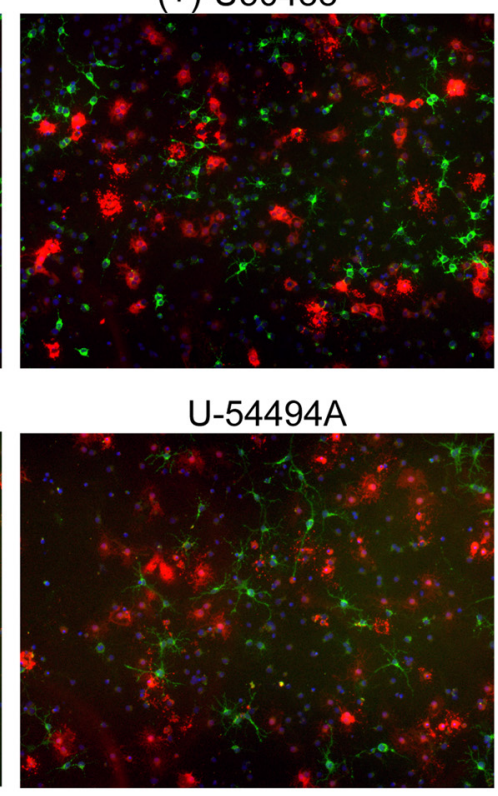

6'-GNTI

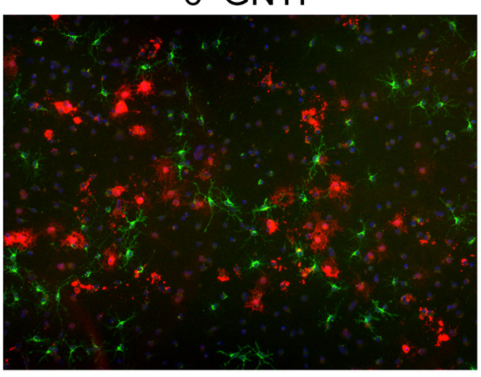

BRL52537
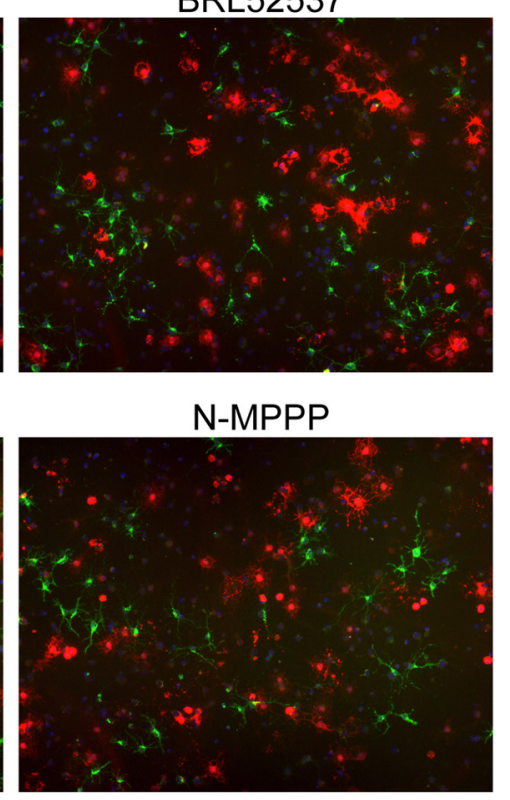

Dynorphin B

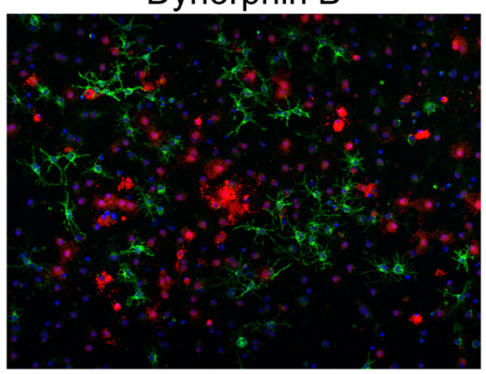

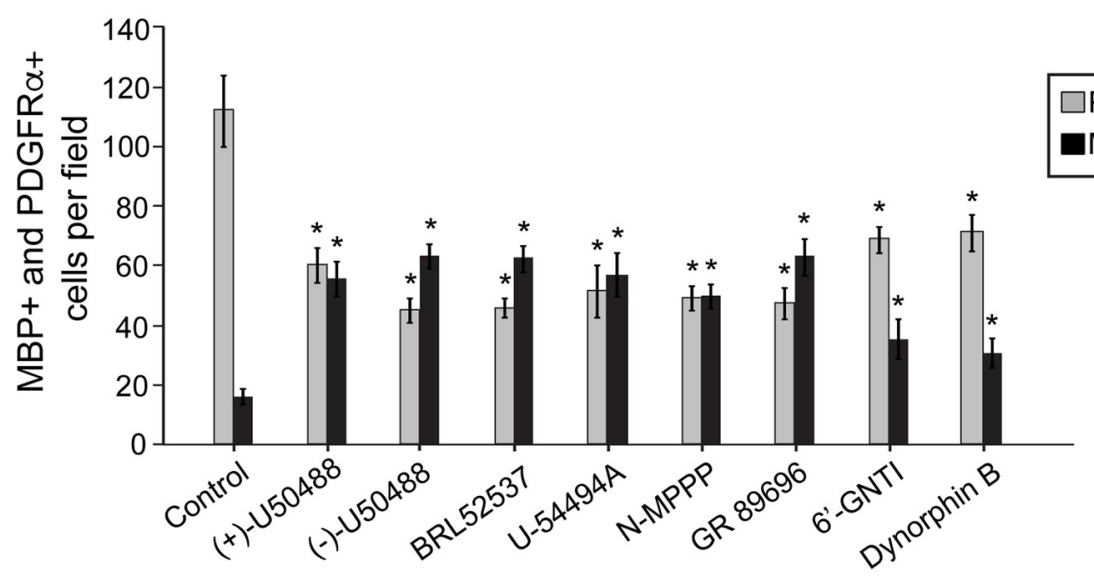

$\square$ PDGFRa

MBP

Figure 2. Validation of KOR agonists using purified OPC cultures. Representative images of rat OPC cultures treated with vehicle (CTL), (+)-U50488, (-)-U50488, BRL52537, U-54494A, N-MPPP,

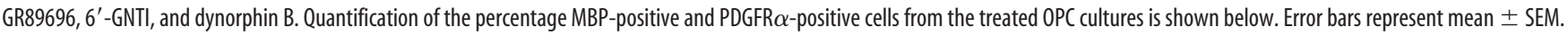
${ }^{*} p<0.05$; significance based on Student's $t$ test. $N=3$ for all experiments.

Fancy et al., 2014). The KOR floxed line was crossed with the Olig2-Cre line to generate conditional knock-out homozygous (Olig2-Cre; KOR $\mathrm{fl} / \mathrm{fl}$ ) and littermate controls (KOR fl/fl). Genotypes of all mice were determined by PCR analysis of tail genomic DNA using appropriate primers. Male and female mice were used for all experiments without bias.

Purification of oligodendrocyte precursor cells. Immunopanning purification of OPCs was performed as previously described (S. Lee et al., 2012). Briefly, OPCs were purified from P7-P9 postnatal rat or mouse brain cortices. Tissue culture dishes were incubated overnight with goat IgG and IgM secondary antibodies to mouse (Jackson Laboratories, Cat- alog \#115-005-004) in $50 \mathrm{~mm}$ Tris-HCl, $\mathrm{pH} 9.5$, at a final concentration of $10 \mu \mathrm{g} / \mathrm{ml}^{-1}$. Dishes were rinsed and incubated at room temperature with primary antibodies for Ran-2, GalC, and O4. Rodent brain hemispheres were diced and dissociated with papain (Worthington) at $37^{\circ} \mathrm{C}$. After trituration, cells were resuspended in a panning buffer $(0.2 \%$ BSA in DPBS) and incubated at room temperature sequentially on the three immunopanning dishes: Ran-2 and GalC were used for negative selection before positive selection with $\mathrm{O} 4$. OPCs were released from the final panning dish using $0.05 \%$ Trypsin (Invitrogen).

Screening compounds with BIMA. The 96 micropillar-well plates were coated with poly-L-lysine $\left(100 \mu \mathrm{g} / \mathrm{ml}^{-1}\right)$ for $1 \mathrm{~h}$, washed twice with 
water and air-dried. In each well, 40,000 rat OPC cells were seeded and maintained in chemically defined medium composed of DMEM (Invitrogen) supplemented with B27 (Invitrogen), N2 (Invitrogen), penicillinstreptomycin (Invitrogen), $\mathrm{N}$-acetylcysteine (Sigma-Aldrich), forskolin (Sigma-Aldrich), and $12.5 \mathrm{ng} / \mathrm{ml}^{-1}$ PDGF-AA (Peprotech) for $2 \mathrm{~d}$ before screening. Compounds $(10 \mathrm{~mm}$ in DMSO; Chemical library, Selleckchem) were diluted to a final concentration of $1 \mu \mathrm{M}$ in the absence of PDGF-AA and added to wells for $3 \mathrm{~d}$ before immunostaining.

Immunofluorescence. Mice were deeply anesthetized with $2.5 \%$ avertin and transcardially perfused with $4 \%$ paraformaldehyde in PBS. Brains and spinal cords were dehydrated in $30 \%$ sucrose and sectioned $(20 \mu \mathrm{m})$ on a cryostat microtome (HM450, Thermo Fisher). OPCs cultured on coverslips were fixed with $4 \%$ paraformaldehyde in PBS. Free-floating sections or coverslips were blocked with $20 \%$ normal goat serum and incubated with primary antibodies overnight at $4^{\circ} \mathrm{C}$, and then incubated with secondary antibodies for $1 \mathrm{~h}$ at room temperature. Primary antibodies included the following: rat monoclonal antibody to MBP (1:500; Millipore, Catalog \#MAB395), rabbit polyclonal antibody to Olig2 (1:500; Millipore, Catalog \#AB9610), rabbit polyclonal antibody to PDGFR $\alpha$ (1:8000; gift from W. B. Stallcup, Sanford Burnham Prebys Medical Discovery Institute, La Jolla, CA), AlexaFluor 488conjugated anti-NG2 (1:400; Millipore, Catalog \#AB5320A4), mouse anti-APC (1:500, Calbiochem, Catalog \#OP80), rabbit anti-NF200 (1: 200, Abcam, Catalog \#Ab 1987), and human anti-myelin oligodendrocyte glycoprotein (MOG; 1:1000, gift from H. C. Von Büdingen, UCSF, San Francisco, CA). Secondary antibodies included the following: AlexaFluor 488-, AlexaFluor 568-, or Cy5-conjugated secondary antibodies to rabbit, mouse, or goat (1:500; Invitrogen). Cell nuclei were identified with DAPI (Vector Laboratories).

Image acquisition and quantification. Fluorescent images from cultured oligodendroglia and cocultures were collected on a Zeiss Axio Imager Z1 fluorescence microscope or a Zeiss LSM-700 confocal microscope with the excitation wavelengths appropriate for AlexaFluor 488 $(488 \mathrm{~nm}), 596(568 \mathrm{~nm}), 647(628 \mathrm{~nm})$, or DAPI $(380 \mathrm{~nm})$. For statistical analysis, at least three representative fields $(20 \times)$ were randomly acquired from each of the wells. Detection and quantification of the rings were performed using Zen software (Zeiss) and Image-Pro Plus software 5.0 (Media Cybernetics). Image acquisition on BIMA (PDGFR $\alpha$ and MBP rings) was scanned using the Zeiss LSM-700 confocal microscope. Images from the screen were acquired at the $z$-position at $10-15 \mathrm{~mm}$ below the tip of the pillar. For statistical analysis, at least three representative fields (100 micropillars) were randomly acquired from each of the wells. Detection and quantification of the rings were performed using Zen software (Zeiss) and Image-Pro Plus software 5.0 (Media Cybernetics).

Electron microscopy. For electron microscopy, animals were perfused through the left ventricle of the heart with $1.25 \%$ glutaraldehyde, $2 \%$ paraformaldehyde in $0.1 \mathrm{~m}$ sodium cacodylate, $\mathrm{pH} 7.4$, after an initial flush with $0.1 \mathrm{~m}$ sodium cacodylate, $\mathrm{pH}$ 7.4. Tissue was then processed at the Electron Microscope Core facilities, Gladstone Institutes, University of California, San Francisco (UCSF). Tissue was postfixed in 2\% osmium tetroxide in the same buffer, en block stained with $2 \%$ aqueous uranyl acetate, dehydrated in acetone, infiltrated, and embedded in LX-112 resin (Ladd Research Industries). Semi-thin sections were stained with toluidine blue. Samples were next ultrathin sectioned on a Reichert Ultracut S ultramicrotome and counter-stained with $0.8 \%$ lead citrate. Grids were examined on a JEOL JEM-1230 transmission electron microscope and photographed with the Gatan Ultrascan 1000 digital camera. $g$-Ratios of myelinated fibers were calculated as the ratio of the diameter of the axon to the diameter of the axon with the myelin sheath, measured using Image-Pro Plus software. Measurements were made on electron micrographs from three pairs of mice.

Oligodendrocyte precursor cell-dorsal root ganglion cocultures. OPCdorsal root ganglion (DRG) cocultures were prepared as previously described (Chong et al., 2012). Briefly, DRG neurons from E15 Sprague-Dawley rats were dissociated, plated (150,000 cells per 25 $\mathrm{mm}$ cover glass), and purified on collagen-coated coverslips in the presence of $100 \mathrm{ng} / \mathrm{ml}^{-} 1 \mathrm{NGF}$ (AbD, Serotec). Neurons were maintained for 3 weeks and washed with DMEM (Invitrogen) extensively to remove any residual NGF before seeding rat or mouse OPCs. Cocultures were grown in chemically defined medium composed of DMEM (Invitrogen) supplemented with B27 (Invitrogen), N2 (Invitrogen), penicillin-streptomycin (Invitrogen), $N$-acetyl-cysteine (Sigma-Aldrich), and forskolin (Sigma-Aldrich).

$R T-P C R$. RNA was extracted from rat OPC cultures or rat corpus callosum using Trizol (Invitrogen, Catalog \#15596026). Reversetranscription was performed using the RETROscript Reverse Transcription Kit (Life Technologies, Catalog \#AM1710). PCR was performed as described previously. Briefly, the DNA polymerase (Invitrogen, Catalog \#10342-020) was used to amplify the target gene of cDNA. Triplicate samples were analyzed for each cDNA with test and internal control primers for the house keeping gene, $\beta$-actin. Primers used for expression analyses are as follows: KORF (tacctgctctcccagtgctt) and KORR (gtt ggttgcggtcttcatct); and ACTINF(cgttgacatccgtaaagacc) and ACTINR (catcgtactcctgcttgct).

Lysolecithin-induced demyelination in the spinal cord. Demyelinated lesions were induced in the corpus callosum of the brain of 8-week-old KOR $\mathrm{cKO}$, control littermates, and wild-type mice. The animals were anesthetized with isofluorane and buprenorphine, and positioned in a stereotaxic frame (Stoelting). A steel needle (33GA, Hamilton) was attached to a $10 \mu \mathrm{l} \mathrm{Ham-}$ ilton syringe through a segment of microtube (PE20, Braintree Scientific) and mounted on a stereotaxic micromanipulator (Harvard Apparatus). Focal demyelination was induced by stereotaxic injection of $1 \mu \mathrm{l}$ of a solution of $1 \%$ LPC (L-a-lysophosphatidylcholine, Sigma-Aldrich) in $0.9 \% \mathrm{NaCl}$. The demyelinating agent was injected unilaterally into the corpus callosum using stereotaxic coordinates of $1.04 \mathrm{~mm}$ posterior to the bregma, $1 \mathrm{~mm}$ lateral, and $1.75 \mathrm{~mm}$ deep from the skull surface. The needle was kept in place for 5 min to reduce reflux along the needle track. After lesioning, mice were administered with $( \pm) \mathrm{U}-50488$ or $10 \%$ DMSO in sterile water (vehicle) by oral gavage $(10 \mathrm{mg} / \mathrm{kg} / \mathrm{d})$ from $0 \mathrm{~d}$ postlesion $(\mathrm{dpl})$ to $10 \mathrm{dpl}$.

iPSC generation and culture. Human induced pluripotent stem cells (iPSCs) were generated from a postnatal patient fibroblasts obtained under approval from UCSF Institutional Review Board. iPSC clones were established by electroporation of a CoMiP episomal vector containing four reprogramming factors (Oct4, Klf4, Sox2, and c-Myc) following a published protocol (Diecke et al., 2015). The patient carried a mutation, which was corrected to wild-type sequence by gene targeting. iPSCs were 
a

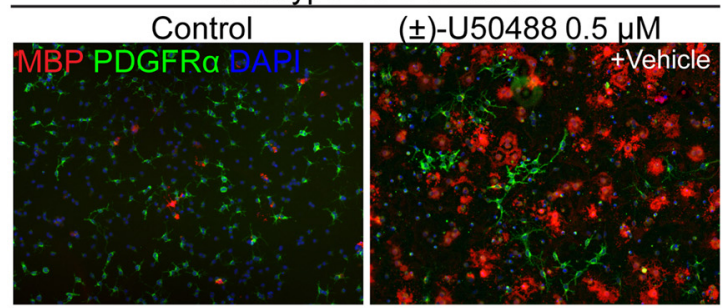

(士)-U50488 $0.5 \mu \mathrm{M}$
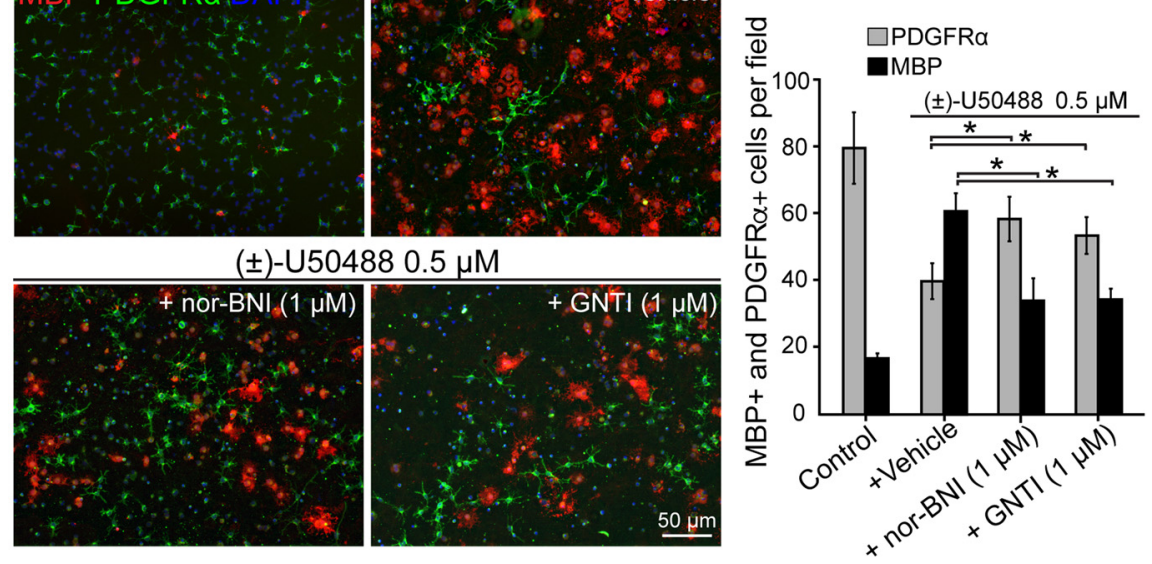

b

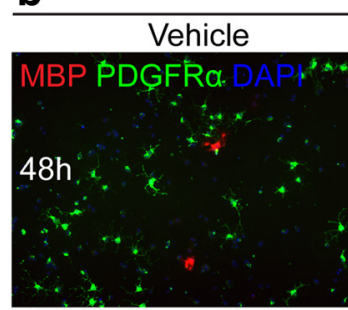

KOR null OPC cultures
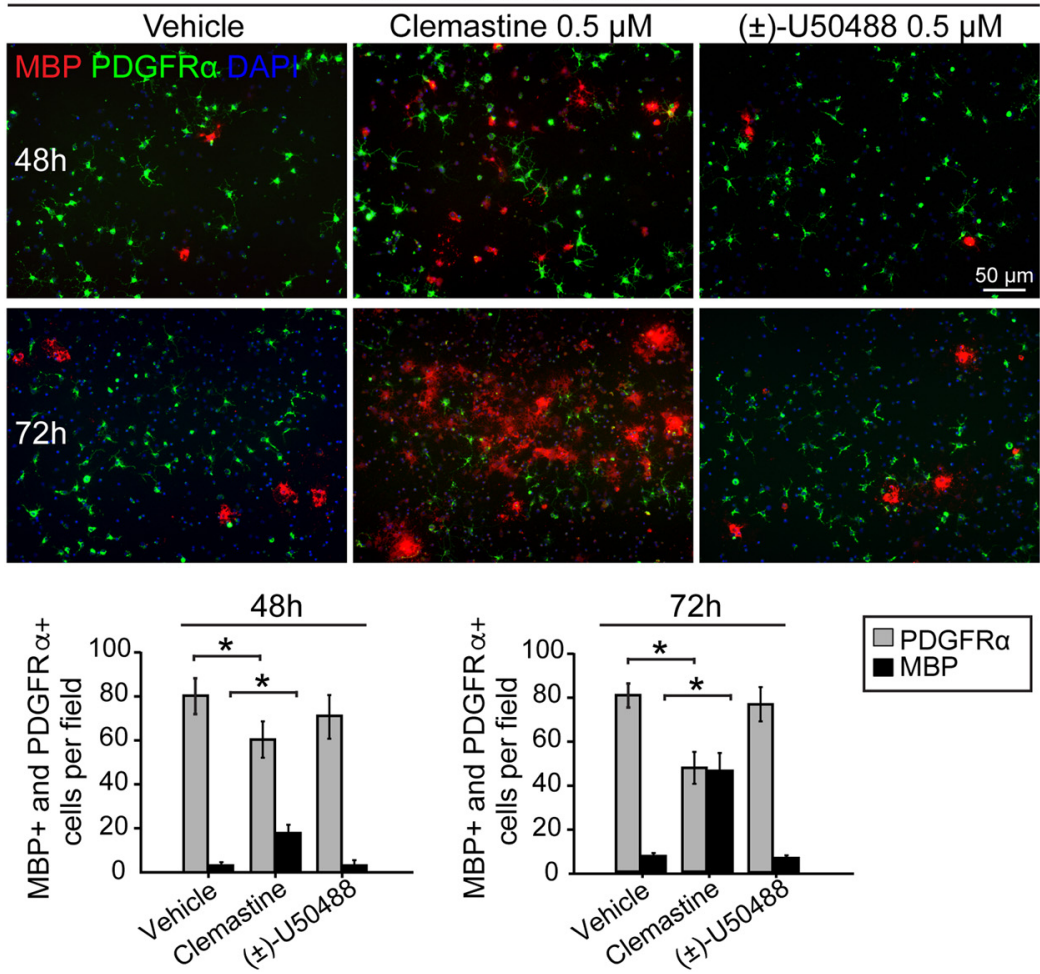

Figure 4. Identification of KOR as the target receptor for ( \pm )-U50488. $\boldsymbol{a}$, Representative images and quantification of purified rat OPCs treated with ( \pm )-U50488 at $0.5 \mu \mathrm{m}$ with or without the presence of KOR-specific antagonists (nor-BNI or GNTI) at $1 \mu \mathrm{M}$ for $48 \mathrm{~h}$ and immunostained for MBP (red) and PDGFR $\alpha$ (green). $\boldsymbol{b}$, Representative images and quantification of purified KOR-null mouse OPCs treated with ( \pm )-U50488 or clemastine at $0.5 \mu \mathrm{m}$ for 48 or $72 \mathrm{~h}$ and immunostained for MBP (red) and PDGFR $\alpha$ (green). Cell nuclei are identified by DAPI (blue). Error bars represent mean \pm SEM. * $p<0.05$; significance based on Student's $t$ test. $N=3$ for all experiments.

maintained on CF1 MEF feeder cells in ES medium containing knockout DMEM/F12, 20\% knock-out serum replacement, nonessential amino acids, Glutamax (Thermo Fisher), and $10 \mathrm{ng} / \mathrm{ml}$ human basic FGF (Peprotech). Pluripotency was confirmed by immunocytochemical staining of Tra1-60 (Cell Signaling Technology) and OCT3/4 (Santa Cruz Biotechnology), as well as teratoma formation and differentiation into ectoderm, mesoderm, and endoderm lineages. Normal karyotype was confirmed. Detailed description of this iPSC will be published elsewhere.

OPC differentiation from iPSC and ( \pm ) U-50488 treatment. A previously published protocol for directed iPSC differentiation to OPC was used with following modifications (Douvaras et al., 2014): human ES medium and human ES medium without basic FGF were used in place of $\mathrm{mTeSR}$ and custom mTeSR, respectively. The concentration of SAG was $0.5 \mu \mathrm{M}$, T3 was $40 \mathrm{ng} / \mathrm{ml}$, and NT3 was $1 \mathrm{ng} / \mathrm{ml}$. Penicillin-streptomycin was omitted from N2 medium, HGF from PDGF medium, and HEPES from glia medium. On day 0 , iPSC were plated at $0.25 \times 10^{6}$ cells/well in a matrigel-coated six-well plate with ES medium without basic FGF, supplemented with dual SMAD inhibitors, RA, and ROCK inhibitor thiazovivin (Santa Cruz Biotechnology). From day 1 to day 4, N2 medium was gradually increased by $25 \%$ each day, reaching $100 \%$ on day 4 . On day 8 , dual SMAD inhibitors were replaced with SAG. On day 12, cells were lifted, dissociated, and seeded on Petri dish for sphere formation. On day 20, medium was changed to PDGF medium. On day 30, spheres were plated on poly-L-ornithine/laminin-coated dishes. On day 45 , medium was changed to glial medium to initiate differentiation. Cells were exposed to $( \pm) \mathrm{U}-50488$ at $1 \mu \mathrm{M}$ from day 45 and analyzed on day 55 .

Statistical analyses. Statistical analyses were performed using two-tailed Student's $t$ test to determine statistical significance that is expressed as ${ }^{\star} p<0.05$ and ${ }^{* *} p<0.01$ compared with control cultures or control mice without compound treatment (vehicle). The investigators were blinded to allocation of compounds during the high-throughput screening until the final statistical analysis.

\section{Results}

GPCRs constitute a large family of receptors that are crucial for numerous cellular processes by transducing extracellular signals into activation of intracellular signaling cascades. GPCRs are therapeutically targeted by $\sim 40 \%$ of all modern medicinal drugs (Overington et al., 2006); therefore, GPCRs are likely targetable receptors for the development of small molecule therapeutics for remyelination. We set out to screen $\sim 250$ bioactive molecules that specifically target GPCRs (SelleckChem), using the high-throughput micropillarscreening platform, BIMA (binary indicant for myelination using micropillar arrays; Mei et al., 2014; Fig. 1a). Micropillars represent freestanding microfibers around which membrane wrapping can be visualized in cross-section, a feature that allows for the detection of "rings" of myelin membrane. The exquisite flexibility in the design of micropillars guarantees consistent uniformity in the experimental approach and any effects from compounds tested can be attributed solely to direct influences on oligodendroglia without indirect effects from neurons (Mei et al., 2014). Purified OPCs were cultured in 96-well plates fabricated with micropillars and were treated with individual compounds at a concentration of $1 \mu \mathrm{M}$ for $3 \mathrm{~d}$ before immunostaining for mature OLs (MBP) and OPCs $(\operatorname{PDGFR} \alpha)$. The effect of each compound was quantified by analyzing MBP (red) and PDGFR $\alpha$ (green) rings from three ran- 
dom 100-micropillar-containing fields by confocal microscopy (Fig. 1b). The data were plotted and grouped into four quadrants based on control compounds. As expected, clemastine, and thyroid hormone (T3), compounds that promote differentiation and myelination, significantly increased the number of MBP-positive rings with a concomitant depletion of $\operatorname{PDGFR} \alpha$-positive rings (Fig. $1 a, b$ ). PDGF, a known mitogen for OPCs, increased the number of PDGFR $\alpha$ rings, while greatly depleting MBP-positive rings (Fig. 1a). Upon completion of screening the GPCR library, we identified a cluster of 12 compounds, namely KOR agonists that significantly enhanced differentiation and wrapping by oligodendrocytes (Fig. 1a). This cluster includes (士)-U50488, (-)-U50488, ICI-199441, U54494, Matrine, N-MPPP, (+)-U50488, BRL52537, GR89696, dynorphin B, $6^{\prime}$-GNTI, and salvinorin A (Fig. $1 a, b$ ). Among all agonists, ( \pm )-U50488 proved to be most effective for oligodendrocyte differentiation (Fig. 1 $a, b$ ). Furthermore, KOR antagonists, nor-binaltorphimine (nor-BNI), DIPPA, GNTI, nalmefene, and naltrexone, exhibited inhibitory effects on oligodendroglial differentiation as shown by decreased MBP rings (Fig. $1 a, b)$. The inverse effects between KOR agonists and antagonists suggest that KOR is an important regulator of oligodendroglial differentiation and myelination. To validate the effects of the KOR agonists identified by BIMA, we tested individual compounds on purified OPC cultures (Figs. 1c, 2). Consistent with findings from BIMA, the KOR agonists promoted OPC differentiation by dramatically increasing $\mathrm{OL}$ numbers $(\mathrm{MBP}+)$ with a concomitant depletion of OPCs (PDGFR $\alpha+$; Figs. $1 c, 2$ ). To test the potential of KOR agonists as candidates that promote myelination, we tested $( \pm)$ U50488 on purified OPC-DRG neuronal cocultures (Chan et al., 2004; Fig. 1d). OPCs were seeded on DRG cultures for 4 d before ( \pm )-U50488 administration. OLs generate multiple "tube-like" myelin segments and ( \pm )-U50488 treatment at 1 $\mu \mathrm{M}$ for $5 \mathrm{~d}$ significantly enhanced myelination in the OPC-DRG cocultures (Fig. 1d). Together, we identified a cluster of KOR agonists that are highly potent in promoting oligodendroglial differentiation and myelination in vitro.

Given that most of the KOR compounds identified are specific to KOR and not other opioid receptor subtypes, we examined KOR expression on purified OPCs by RT-PCR and immunostaining (Fig. 3). KOR mRNA was detected in the corpus callosum and purified OPC cultures (Fig.
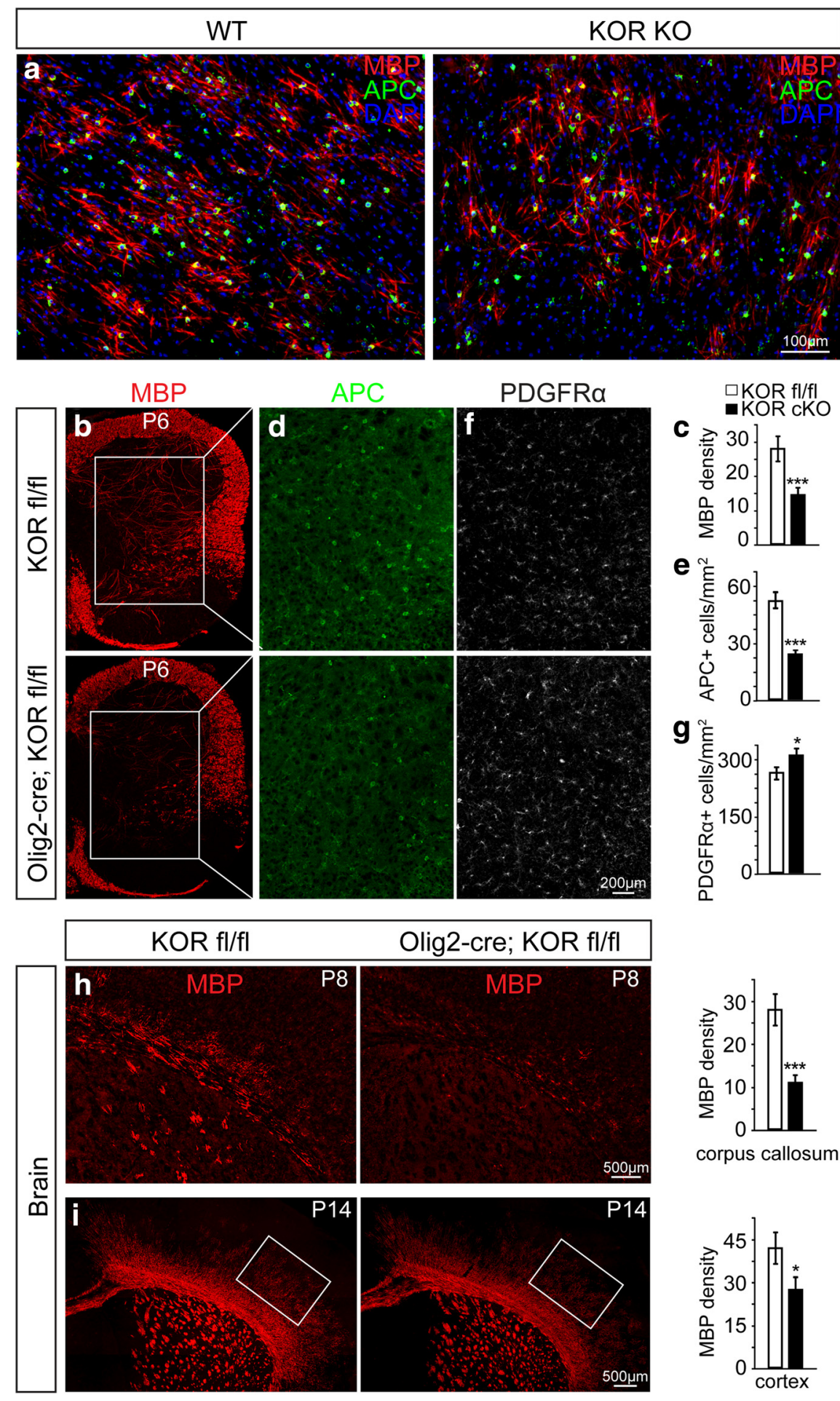

corpus callosum

Figure 5. KOR deletion in OPCs delays myelination in the developing CNS. a, Cocultures of wild-type (WT) and KOR-null (KO) mouse OPCs on DRGs. $\boldsymbol{a}$, Representative images of MBP (red), APC (green), and DAPI (blue) immunostaining after 11 DIV. $\boldsymbol{b}, \boldsymbol{d}, \boldsymbol{f}$, Representative images of MBP $(\boldsymbol{b}), \operatorname{APC}(\boldsymbol{d})$, and PDGFR $\alpha(\boldsymbol{f})$ immunostaining in the spinal cord of WT and KOR cK0 mice at P6. Boxes in $\boldsymbol{b}$ show regions analyzed in $\boldsymbol{d}$ and $\boldsymbol{f}$. $\boldsymbol{c}, \boldsymbol{e}, \boldsymbol{g}$, Quantification of $\operatorname{MBP}(\boldsymbol{c}), \operatorname{APC}(\boldsymbol{e})$, and PDGFR $\alpha(\boldsymbol{g})$ immunostaining shows significant differences between the groups. $\boldsymbol{h}, \boldsymbol{i}, \mathrm{MBP}$ immunostaining and quantification in the corpus callosum at P8 (h), and cortex at P14 (i) of wild-type and KOR cKO mice. Boxes in $i$ show regions of cortex quantified. Error bars represent mean \pm SEM. ${ }^{*} p<0.05,{ }^{* * *} p<0.001$; significance based on Student's $t$ test. $N=3$ for all experiments.

$3 a)$. OPCs expressed the KOR in processes and cell bodies, suggesting that KOR may play a role during oligodendroglial development (Fig. 3b). To demonstrate specificity, we investigated whether KOR inhibition could counteract the effect of agonist 

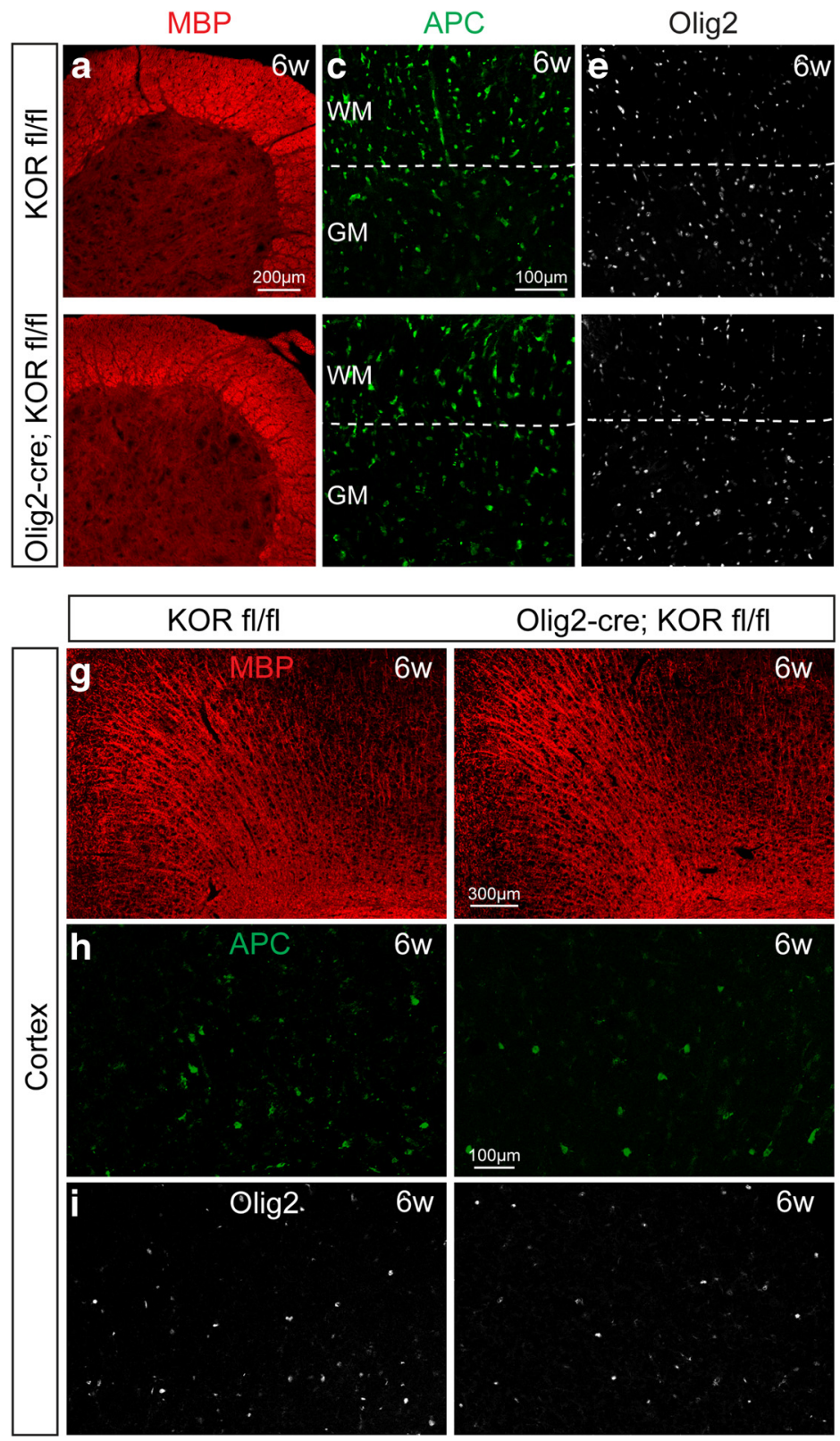

Figure 6. Myelination delay in KOR cKOs catches up in adults. $\boldsymbol{a}, \boldsymbol{c}, \boldsymbol{e}$, Representative images of $\operatorname{MBP}(\boldsymbol{a}), \operatorname{APC}(\boldsymbol{c})$, and Olig2 (e) immunostaining in the spinal cord of control and KOR cKO mice at 6 weeks of age. Dotted lines in $c$ and $e$ separate white matter (WM; top) and gray matter (GM; bottom). $\boldsymbol{b}, \boldsymbol{d}, \boldsymbol{f}$, Quantification of MBP (b), APC (d), and Olig2 (f) immunostaining in gray matter shows no differences between the groups. $\boldsymbol{g}-\boldsymbol{i}$, Representative images of $\operatorname{MBP}(\boldsymbol{g}), \operatorname{APC}(\boldsymbol{h})$, and 0lig2 (i) immunostaining and quantification in the cortex of control and KOR cKO mice at 6 weeks of age. Error bars represent mean \pm SEM. $N=3$ for all experiments.

treatment. We exposed purified wild-type OPCs to (土)-U50488 with or without the presence of KOR-specific antagonists (Fig. 4a). ( \pm )-U50488 significantly enhanced OPC differentiation at a concentration of $0.5 \mu \mathrm{M}$. This effect was significantly abolished by KOR-specific antagonists (nor-BNI or GNTI at $1 \mu \mathrm{M}$ ) compared with the vehicle control (Fig. 4a). To determine whether KOR is the receptor that mediates the effect of $( \pm)$-U50488, OPCs were purified from KOR knock-out mice. The number of MBP+ OLs and PDGFR $\alpha+$ OPCs were quantified after treatment with clemastine or ( \pm )-U50488 for 48 or $72 \mathrm{~h}$ (Fig. $4 b$ ). Clemastine was used as a positive control to ensure that the KOR knock-out OPCs retained their potential to differentiate. As expected, OL $(\mathrm{MBP}+)$ numbers were greatly increased with a simultaneous

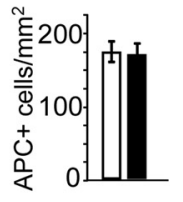

पKOR fl/fl

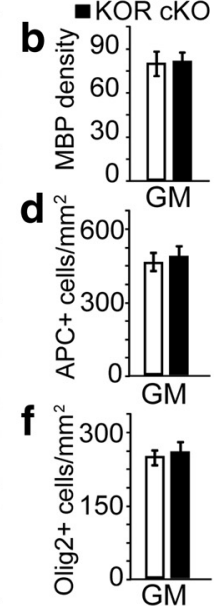

$\square K O R$
fl/fl
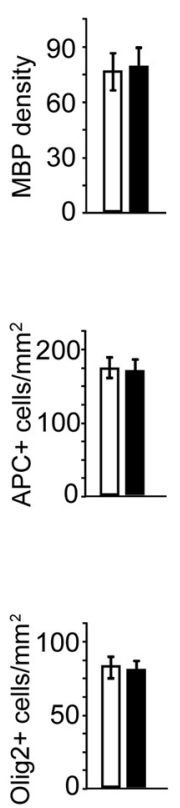
oping CNS.

decrease in the number of OPCs $(\mathrm{PDGFR} \alpha+)$ with clemastine treatment, suggesting that KOR-null OPCs are intrinsically capable of differentiating into mature OLs in vitro (Fig. $4 b$ ). Interestingly, KOR knock-out OPCs did not respond to ( \pm )-U50488, resulting in similar numbers of OPCs (PDGFR $\alpha+$ ) and OLs $(\mathrm{MBP}+)$ compared with vehicle control, indicating that KOR is the sole receptor mediating the effect of ( \pm )-U50488 on oligodendroglial differentiation (Fig. $4 b$ ).

To explore the role of KOR in regulating oligodendroglial differentiation and myelination we compared the differentiation of WT and KOR-null OPCs in cocultures. After $11 \mathrm{~d}$ we saw what appeared to be a delay in differentiation and myelination in the $\mathrm{KO}$ cultures (Fig. 5a). We decided to examine this in vivo during development. We crossed a floxed KOR (KOR fl/fl) mouse line with the Olig2-Cre driver to induce conditional deletion of KOR in OPCs (Olig2-Cre; KOR fl/fl). Because KOR agonism promotes oligodendroglial differentiation and myelination in vitro, we hypothesized that KOR positively regulates the differentiation and myelination of oligodendroglia in the developing CNS. Initially, myelination in the P6 mouse spinal cords was examined (Fig. $5 b-g$ ), and the myelin sheaths are significantly less dense with a decrease of myelin density by $50 \%$ in the gray matter in KOR cKO mice (Olig2-Cre; $\mathrm{KOR} \mathrm{fl} / \mathrm{fl}$ ) compared with littermate wild-type mice (KOR fl/fl; Fig. 5b,c), with a corresponding significant decrease in the number of OLs (Fig. $5 d, e$ ) and an increase of PDGFR $\alpha+$ OPC numbers (Fig. 5f,g). To extend these observations into the brain, we assessed early myelin development along white matter tracts. Consistent with spinal cord analyses, an obvious delay of myelin development was observed in the corpus callosum of KOR cKO mice compared with wild-type mice at $\mathrm{P} 8$ (Fig. 5h). The myelin development in KOR cKO mice quickly catches up in the corpus callosum by P14 (Fig. 5i), but remains delayed in the cortex (Fig. 5j). By 6 weeks, the myelin density in spinal cord (Fig. $6 a, b)$ and cortex (Fig. $6 g$ ) was not found significantly different between KOR $\mathrm{cKO}$ and wild-type mice. Mature oligodendrocyte (APC+) and total oligodendrocyte (Olig2 + ) densities also appeared similar between KOR cKO and wild-type mice by 6 weeks (Fig. $6 c-f, h, i)$. These results indicate KOR as a positive regulator for oligodendroglia differentiation and myelination in the devel-

To evaluate the effect of $( \pm)$-U50488 and the role of KOR on the kinetics of remyelination, we used the lysolecithin- 
a

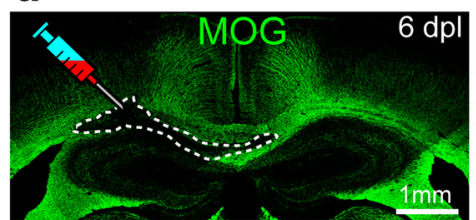

KOR fl/fl

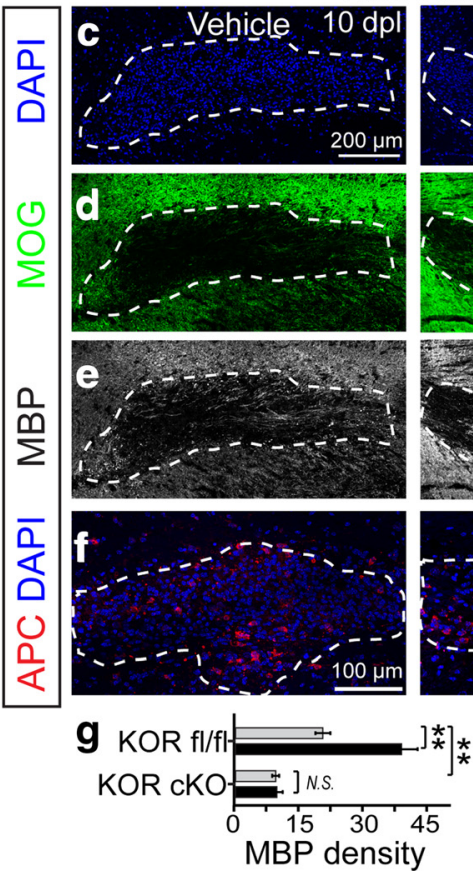

Vehicle

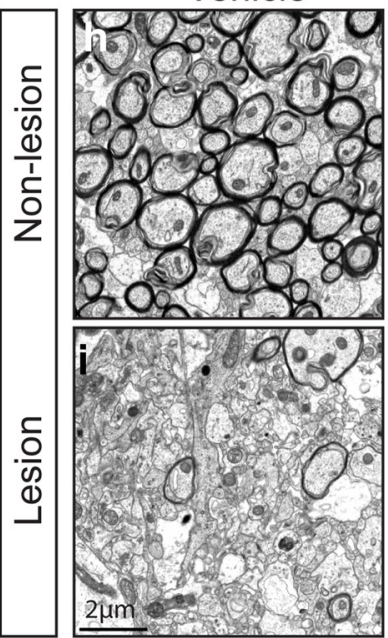

b $\quad$ KOR fl/fl
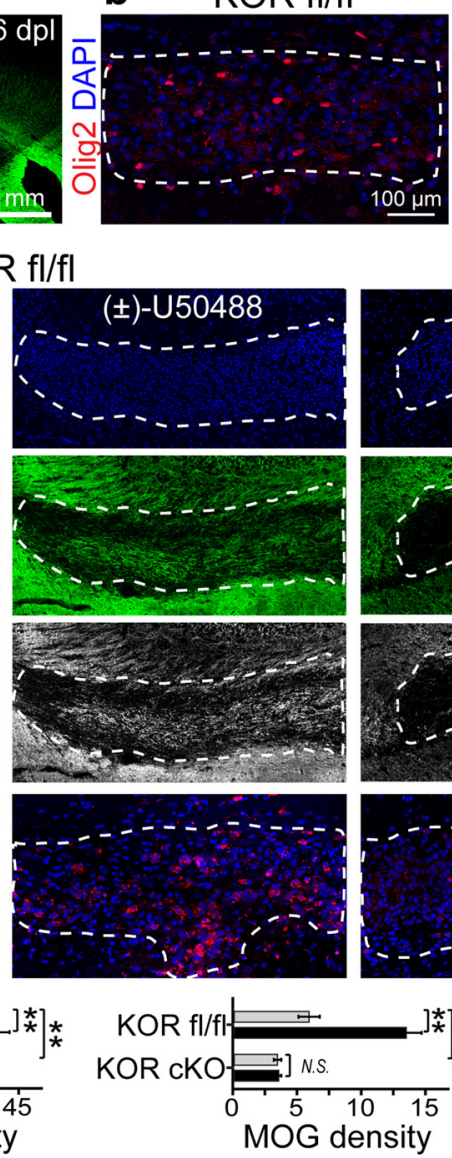

Olig2-cre; KOR fl/fl

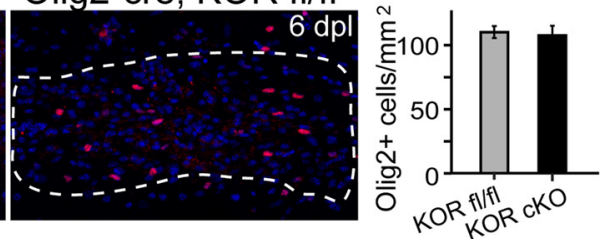

Olig2-cre; KOR fl/fl
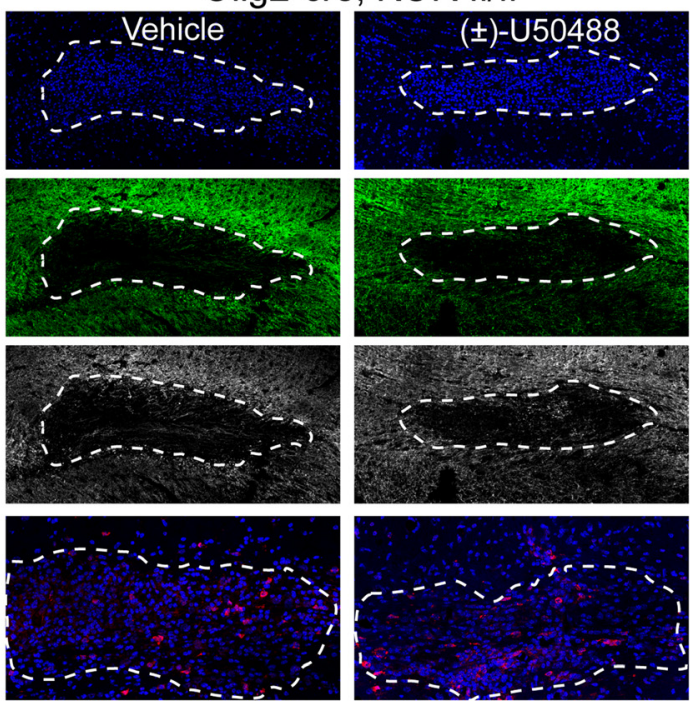

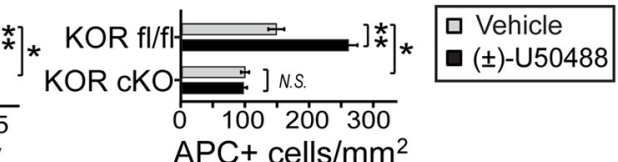

(士)-U50488
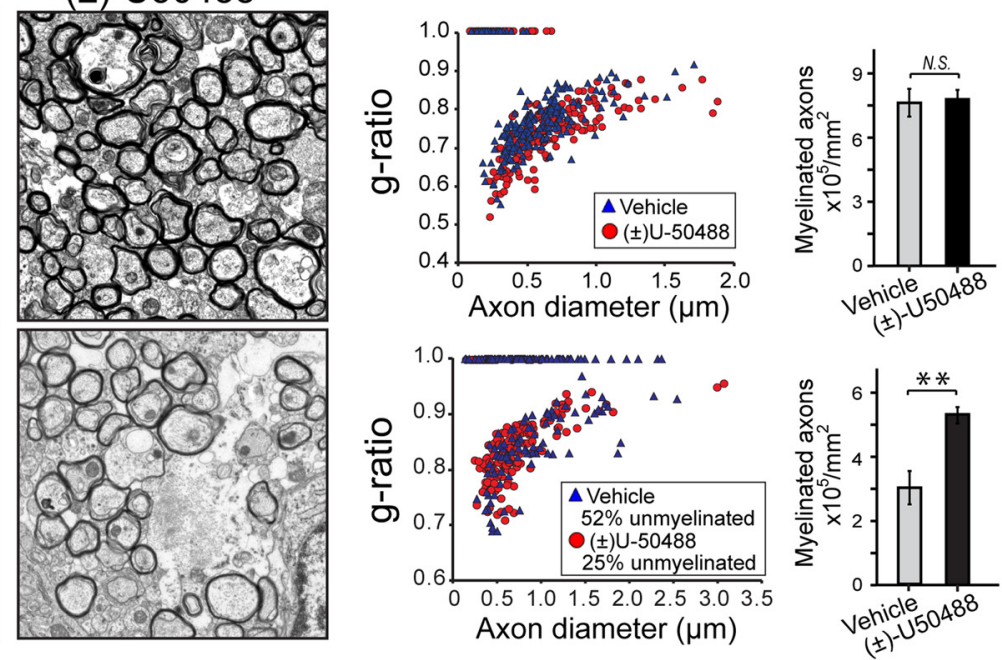

Figure 7. ( \pm )-U50488 accelerates remyelination via KOR on oligodendroglia in the lysolecithin-induced demyelination model. $\boldsymbol{a}, \mathrm{MOG}$ staining displays focal demyelination (outlined area) in mouse corpus callosum at $6 \mathrm{dpl}$. b, Representative images and quantification of OPC recruitment in the lesions of KOR cKO and control mice at $6 \mathrm{dpl}$. Olig2 (red) identifies recruited OPCs and DAPI (blue) identifies cell nuclei. $\boldsymbol{c}$, Lesions were identified by the accumulated nuclei (DAPI, blue) and outlined. Brains were analyzed by immunostaining for MOG (d), MBP $(\boldsymbol{e})$, and APC $(\boldsymbol{f})$ at $10 \mathrm{dpl}$ in KOR cKO and control mice treated with or without ( \pm )-U50488 via oral gavage at $10 \mathrm{mg} / \mathrm{kg} / \mathrm{d}$ from 0 dpl. $\boldsymbol{g}$, Quantification of MBP and MOG density and APC + cell numbers in the lesions. $\boldsymbol{h}, \boldsymbol{i}$, Remyelination was analyzed at $14 \mathrm{dpl}$ using electron microscopy in wild-type mice treated with vehicle or ( \pm )-U50488 via oral gavage from 0 dpl in non-lesioned areas (h) and in the lesions (i). Quantification of myelin sheath thickness and the proportion of myelinated and unmyelinated axons are shown on the right. The scatterplot displays $g$-ratios of individual axons as a function of axonal diameter. Error bars represent mean \pm SEM. ${ }^{*} p<0.05,{ }^{* *} p<0.01$; significance based on Student's $t$ test. $N=3$ for all experiments.

induced demyelination mouse model (Fancy et al., 2011, 2014; Chefer et al., 2013). Demyelination was induced by injecting 1 $\mu \mathrm{l}$ of $1 \%$ lysolecithin solution at the coordinates: AP: -1.04 , ML: 1 , and DV: -1.75 from bregma into the corpus callosum of 8 -week-old mice. Indicative of this demyelinating model is the timeline for repair, comprising active demyelination (1-3 $\mathrm{d}$ postlesion $(\mathrm{dpl})$ ), OPC recruitment (3-7 dpl, peaking at 5 $\mathrm{dpl}$ ), oligodendrocyte differentiation (7-10 dpl) and active remyelination (14-21 dpl; Chong et al., 2012; Mei et al., 2014). MOG immunostaining clearly illustrates the demyelinating 

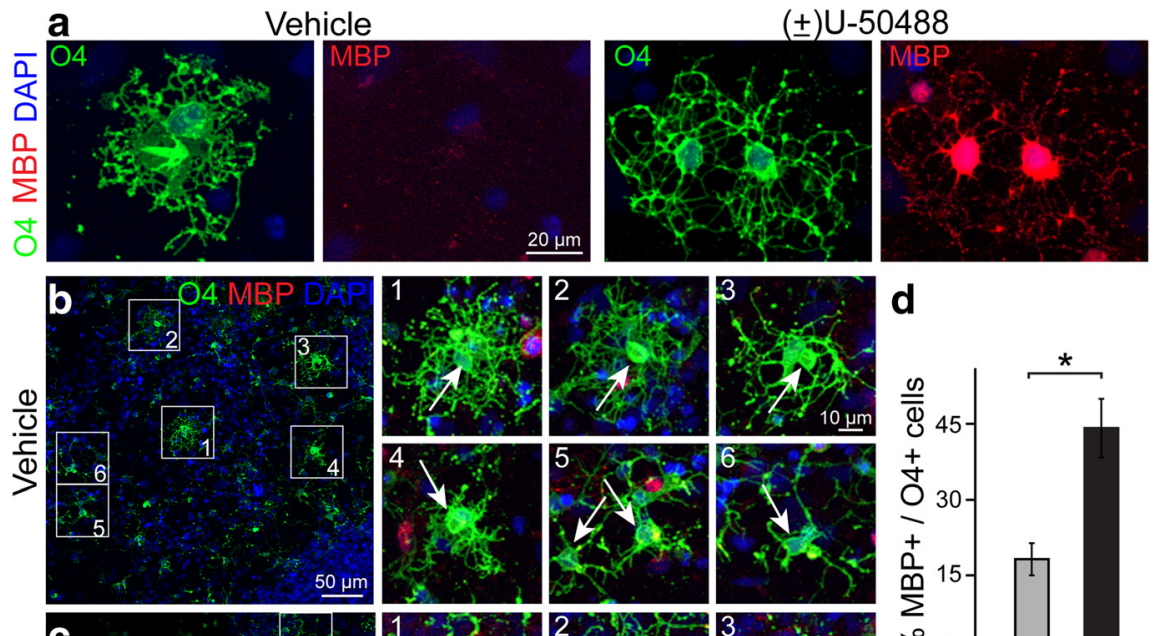

d
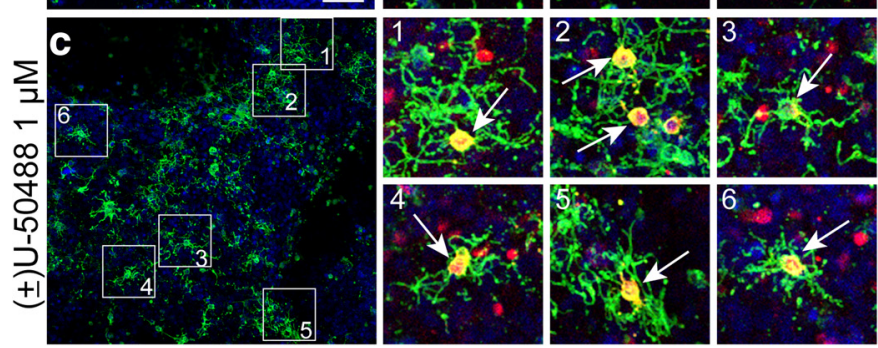

Figure 8. ( \pm )-U50488 enhances the maturation of human iPSC-derived oligodendroglia. Human iPSC-derived oligodendroglia were treated with vehicle or ( \pm )-U50488 at $1 \mu \mathrm{m}$ for $10 \mathrm{~d}$ and immunostained with markers for immature oligodendrocytes (04; green), differentiation (MBP; red), and cell nuclei (DAPI; blue). $\boldsymbol{a}$, High-magnification images of human iPSC-derived oligodendroglia showing faint MBP immunostaining in vehicle-treated cells while ( \pm )-U50488-treated cells show intense MBP immunostaining and elaborate MBP + membranes. $\boldsymbol{b}$ - $\boldsymbol{d}$, Representative fields of human iPSC-derived oligodendroglia treated with vehicle $(\boldsymbol{b})$ or ( \pm )-U50488 (c) and quantification of the percentage of $04+$ cells that are MBP $+(\boldsymbol{d})$. Error bars represent mean \pm SEM. ${ }^{*} p<0.05$; significance based on Student's $t$ test. $N=3$ for all experiments.

lesion induced by lysolecithin in the corpus callosum at $6 \mathrm{dpl}$ (Fig. 7a). To investigate the recruitment or proliferation of OPCs upon KOR deletion, we examined the number of Olig2positive cells in the lesions at $6 \mathrm{dpl}$, which were outlined by the accumulation of nuclei (DAPI). We did not find a significant change between KOR conditional knock-out (cKO) and control (KOR fl/fl) mice (Fig. $7 b$ ), suggesting that KOR deletion does not effect OPC proliferation or migration. To investigate the role of KOR on remyelination, $( \pm) \mathrm{U}-50488$ or vehicle was administered by oral gavage $(10 \mathrm{mg} / \mathrm{kg} / \mathrm{d})$ from $0 \mathrm{dpl}$ to $\mathrm{KOR}$ $\mathrm{cKO}$ and littermate controls (KOR fl/fl), and oligodendroglial differentiation was quantified at $10 \mathrm{dpl}$ (Fig. $7 c-g$ ). Immunostaining for MBP and MOG displayed enhanced myelin protein expression in the lesion of control mice treated with $( \pm) \mathrm{U}-50488$ compared with vehicle, likely due to accelerated oligodendroglial differentiation, as illustrated by the increase in mature OL $(\mathrm{APC}+)$ cell number after $( \pm) \mathrm{U}-50488$ treatment (Fig. $7 c-g$ ). In the KOR cKO mice, oligodendroglia differentiation was equally inhibited in the lesions treated with either $( \pm)$ U-50488 or vehicle control (Fig. $7 c-g$ ), indicating that KOR is an important regulator for oligodendroglial differentiation and also the target receptor that mediates the effect of $( \pm) \mathrm{U}-50488$ on remyelination after toxic demyelination (Fig. $7 c-g$ ). Interestingly, there also seemed to be less remyelination in vehicle-treated $\mathrm{KOR}$ cKO mice versus vehicle-treated controls (Fig. $7 c-g$ ). This suggests that KORs play a role during remyelination as in development as shown previously. We analyzed the effect of $( \pm) \mathrm{U}-50488$ on remyelination in wild-type mice at $14 \mathrm{dpl}$ by transmission electron microscopy with $( \pm) \mathrm{U}-50488$ or vehicle treatment from $0 \mathrm{dpl}$

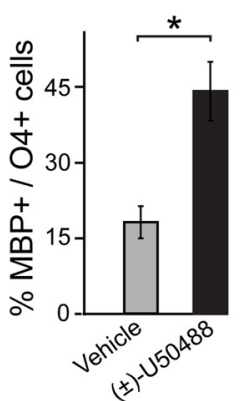

(Fig. $7 h, i)$. In the non-lesioned areas, $( \pm) \mathrm{U}-50488$ treatment did not alter the $g$-ratio nor the myelinated axon density compared with the vehicle, indicating ( \pm U-50488 administration does not result in abnormal myelination (Fig. $7 h)$. In the lesioned areas, remyelination is identified by thinner myelin sheaths with an increased $g$-ratio (Fig. 7i). $( \pm) \mathrm{U}-50488$ treatment greatly increased the number of remyelinated axons in the lesions compared with the vehicle control, with a concomitant decrease in the number of unmyelinated axons (Fig. 7i). These findings further support the conclusion that $( \pm) \mathrm{U}$ 50488 greatly promotes remyelination after toxic demyelination via KOR on OPCs.

To determine whether these findings are relevant to human oligodendrocytes, human iPSC-derived OPCs were examined to determine whether agonism of the KOR could be a potential and effective therapeutic approach for clinical use (Wang et al., 2013; Yang et al., 2013; Douvaras et al., 2014; Fossati and Douvaras, 2014). We treated human iPSC-derived OPCs with $( \pm) \mathrm{U}-50488$ at $1 \mu \mathrm{M}$ for $10 \mathrm{~d}$ and immunostained for $\mathrm{O} 4$ and $\mathrm{MBP}$ (Fig. 8). Immature oligodendroglial cells express $\mathrm{O} 4$ with multiple elaborated processes and initiate MBP expression upon terminal maturation (Fig. 8a). Exposure to ( \pm ) U-50488 resulted in a significant increase in the ratio of $\mathrm{MBP}+$ cells to $\mathrm{O} 4$-positive cells compared with the vehicle control (Fig. $8 b-d$ ), indicating that agonism of KOR enhanced maturation of human oligodendroglial cells in vitro.

\section{Discussion}

Remyelination therapy promises to be an effective approach, because achieving even partial functional recovery from demyelinating diseases is clearly an unmet need. Increasing evidence indicates that myelin is important for maintaining axonal integrity, presumably by providing physical and metabolic support to axons (Lappe-Siefke et al., 2003; Fünfschilling et al., 2012; Y. Lee et al., 2012; Morrison et al., 2013; Saab et al., 2013). Recent efforts with high-throughput screening have identified a number of compounds that enhance remyelination (Deshmukh et al., 2013; Mei et al., 2014; Najm et al., 2015). However, the clinical potential for these drugs are limited by deleterious side effects and/or offtarget receptors. Continued screening of novel and existing small molecule libraries combined with medicinal chemistry should greatly reduce side effects and improve potency. Our screening efforts with a GPCR library identified a cluster of compounds that greatly enhance oligodendroglial differentiation including the KOR agonist, $( \pm) \mathrm{U}-50488$, which enhanced remyelination in lysolecithin-induced lesions and underscores KOR agonism as a means to accelerate remyelination.

Consistent with our findings, a recent study showed that $( \pm)$ U-50488 treatment was capable of alleviating the clinical score of EAE mice, an inflammatory demyelination model, and protected myelin from breakdown in the cuprizone demy- 
elination model (Du et al., 2016). However, cell autonomy of KOR effects remains unclear and the potential for KOR agonism to influence inflammation remains a possibility. Through the use of oligodendroglial-specific KOR-null mice our results demonstrate that KOR agonism specifically in oligodendroglia is required for the myelination-promoting effects of $( \pm)$ U-50488 treatment. Furthermore, our data indicate that KOR acts as a positive regulator of myelination during both development and remyelination. Moreover, to translate the findings into potential clinical therapeutics, it is important to understand whether human OPCs respond to KOR agonists similar to rodent OPCs. Our results indicate that KOR agonists promote the differentiation of human iPSC-derived OPCs, suggesting that KOR agonism is likely to be an effective strategy for remyelination therapy in humans.

KOR is widely expressed throughout the CNS. Selective activation of KOR produces anti-nociception in animal models without a risk of physical dependence or respiratory failure (Millan et al., 1989). However, it is notable that, in addition to the analgesic effect, KOR activation has been shown to have a number of undesirable side effects including dysphoria (Land et al., 2008) and sedation (Dykstra et al., 1987). These side effects might dampen the therapeutic potential of KOR agonists in the treatment of demyelinating diseases. Thus, a redesign or structural modification of KOR agonists to diminish side effects and improve potency may overcome this hurdle in the future (Zhou et al., 2013).

All together, we identify a cluster of KOR agonists that are highly potent in promoting oligodendroglial differentiation and myelination and KOR as the mediator of these effects. Our results further demonstrate a role for KOR agonism in promoting remyelination and offer a promising target for future remyelinating therapies.

\section{References}

Chan JR, Watkins TA, Cosgaya JM, Zhang C, Chen L, Reichardt LF, Shooter EM, Barres BA (2004) NGF controls axonal receptivity to myelination by Schwann cells or oligodendrocytes. Neuron 43:183-191. CrossRef Medline

Chang A, Nishiyama A, Peterson J, Prineas J, Trapp BD (2000) NG2positive oligodendrocyte progenitor cells in adult human brain and multiple sclerosis lesions. J Neurosci 20:6404-6412. Medline

Chefer VI, Bäckman CM, Gigante ED, Shippenberg TS (2013) Kappa opioid receptors on dopaminergic neurons are necessary for kappa-mediated place aversion. Neuropsychopharmacology 38:2623-2631. CrossRef Medline

Chong SY, Rosenberg SS, Fancy SP, Zhao C, Shen YA, Hahn AT, McGee AW, Xu X, Zheng B, Zhang LI, Rowitch DH, Franklin RJ, Lu QR, Chan JR (2012) Neurite outgrowth inhibitor Nogo-A establishes spatial segregation and extent of oligodendrocyte myelination. Proc Natl Acad Sci U S A 109:1299-1304. CrossRef Medline

Deshmukh VA, Tardif V, Lyssiotis CA, Green CC, Kerman B, Kim HJ, Padmanabhan K, Swoboda JG, Ahmad I, Kondo T, Gage FH, Theofilopoulos AN, Lawson BR, Schultz PG, Lairson LL (2013) A regenerative approach to the treatment of multiple sclerosis. Nature 502:327-332. CrossRef Medline

Diecke S, Lu J, Lee J, Termglinchan V, Kooreman NG, Burridge PW, Ebert AD, Churko JM, Sharma A, Kay MA, Wu JC (2015) Novel codonoptimized mini-intronic plasmid for efficient, inexpensive, and xeno-free induction of pluripotency. Sci Rep 5:8081. CrossRef Medline

Douvaras P, Wang J, Zimmer M, Hanchuk S, O’Bara MA, Sadiq S, Sim FJ, Goldman J, Fossati V (2014) Efficient generation of myelinating oligodendrocytes from primary progressive multiple sclerosis patients by induced pluripotent stem cells. Stem Cell Reports 3:250-259. CrossRef Medline

Du C, Duan Y, Wei W, Cai Y, Chai H, Lv J, Du X, Zhu J, Xie X (2016) Kappa opioid receptor activation alleviates experimental autoimmune encepha- lomyelitis and promotes oligodendrocyte-mediated remyelination. Nat Commun 7:11120. CrossRef Medline

Dykstra LA, Gmerek DE, Winger G, Woods JH (1987) Kappa opioids in rhesus monkeys: I. Diuresis, sedation, analgesia and discriminative stimulus effects. J Pharmacol Exp Ther 242:413-420. Medline

Fancy SP, Harrington EP, Yuen TJ, Silbereis JC, Zhao C, Baranzini SE, Bruce CC, Otero JJ, Huang EJ, Nusse R, Franklin RJ, Rowitch DH (2011) Axin2 as regulatory and therapeutic target in newborn brain injury and remyelination. Nat Neurosci 14:1009-1016. CrossRef Medline

Fancy SP, Harrington EP, Baranzini SE, Silbereis JC, Shiow LR, Yuen TJ, Huang EJ, Lomvardas S, Rowitch DH (2014) Parallel states of pathological Wnt signaling in neonatal brain injury and colon cancer. Nat Neurosci 17:506-512. CrossRef Medline

Fossati V, Douvaras P (2014) Generating induced pluripotent stem cells for multiple sclerosis therapy. Regen Med 9:709-711. CrossRef Medline

Fünfschilling U, Supplie LM, Mahad D, Boretius S, Saab AS, Edgar J, Brinkmann BG, Kassmann CM, Tzvetanova ID, Möbius W, Diaz F, Meijer D, Suter U, Hamprecht B, Sereda MW, Moraes CT, Frahm J, Goebbels S, Nave KA (2012) Glycolytic oligodendrocytes maintain myelin and long-term axonal integrity. Nature 485:517-521. Medline

Keirstead HS, Levine JM, Blakemore WF (1998) Response of the oligodendrocyte progenitor cell population (defined by NG2 labelling) to demyelination of the adult spinal cord. Glia 22:161-170. CrossRef Medline

Land BB, Bruchas MR, Lemos JC, Xu M, Melief EJ, Chavkin C (2008) The dysphoric component of stress is encoded by activation of the dynorphinopioid system. J Neurosci 28:407-414. CrossRef Medline

Lappe-Siefke C, Goebbels S, Gravel M, Nicksch E, Lee J, Braun PE, Griffiths IR, Nave KA (2003) Disruption of Cnp1 uncouples oligodendroglial functions in axonal support and myelination. Nat Genet 33:366-374. CrossRef Medline

Lee S, Leach MK, Redmond SA, Chong SY, Mellon SH, Tuck SJ, Feng ZQ, Corey JM, Chan JR (2012) A culture system to study oligodendrocyte myelination processes using engineered nanofibers. Nat Methods 9:917922. CrossRef Medline

Lee Y, Morrison BM, Li Y, Lengacher S, Farah MH, Hoffman PN, Liu Y, Tsingalia A, Jin L, Zhang PW, Pellerin L, Magistretti PJ, Rothstein JD (2012) Oligodendroglia metabolically support axons and contribute to neurodegeneration. Nature 487:443-448. CrossRef Medline

Mei F, Fancy SP, Shen YA, Niu J, Zhao C, Presley B, Miao E, Lee S, Mayoral SR, Redmond SA, Etxeberria A, Xiao L, Franklin RJ, Green A, Hauser SL, Chan JR (2014) Micropillar arrays as a high-throughput screening platform for therapeutics in multiple sclerosis. Nat Med 20:954960. CrossRef Medline

Millan MJ, Członkowski A, Lipkowski A, Herz A (1989) Kappa-opioid receptor-mediated antinociception in the rat: II. Supraspinal in addition to spinal sites of action. J Pharmacol Exp Ther 251:342-350. Medline

Morrison BM, Lee Y, Rothstein JD (2013) Oligodendroglia: metabolic supporters of axons. Trends Cell Biol 23:644-651. CrossRef Medline

Najm FJ, Madhavan M, Zaremba A, Shick E, Karl RT, Factor DC, Miller TE, Nevin ZS, Kantor C, Sargent A, Quick KL, Schlatzer DM, Tang H, Papoian R, Brimacombe KR, Shen M, Boxer MB, Jadhav A, Robinson AP, Podojil JR, et al. (2015) Drug-based modulation of endogenous stem cells promotes functional remyelination in vivo. Nature 522:216-220. CrossRef Medline

Nunes MC, Roy NS, Keyoung HM, Goodman RR, McKhann G 2nd, Jiang L, Kang J, Nedergaard M, Goldman SA (2003) Identification and isolation of multipotential neural progenitor cells from the subcortical white matter of the adult human brain. Nat Med 9:439-447. CrossRef Medline

Overington JP, Al-Lazikani B, Hopkins AL (2006) How many drug targets are there? Nat Rev Drug Discov 5:993-996. CrossRef Medline

Saab AS, Tzvetanova ID, Nave KA (2013) The role of myelin and oligodendrocytes in axonal energy metabolism. Curr Opin Neurobiol 23:10651072. CrossRef Medline

Scolding N, Franklin R, Stevens S, Heldin CH, Compston A, Newcombe J (1998) Oligodendrocyte progenitors are present in the normal adult human CNS and in the lesions of multiple sclerosis. Brain 121:2221-2228. CrossRef Medline 
Wang S, Bates J, Li X, Schanz S, Chandler-Militello D, Levine C, Maherali N, Studer L, Hochedlinger K, Windrem M, Goldman SA (2013) Human iPSC-derived oligodendrocyte progenitor cells can myelinate and rescue a mouse model of congenital hypomyelination. Cell Stem Cell 12:252-264. CrossRef Medline

Wolswijk G (1998) Chronic stage multiple sclerosis lesions contain a relatively quiescent population of oligodendrocyte precursor cells. J Neurosci 18:601-609. Medline

Woodruff RH, Fruttiger M, Richardson WD, Franklin RJ (2004) Plateletderived growth factor regulates oligodendrocyte progenitor numbers in adult CNS and their response following CNS demyelination. Mol Cell Neurosci 25:252-262. CrossRef Medline
Yang N, Zuchero JB, Ahlenius H, Marro S, Ng YH, Vierbuchen T, Hawkins JS, Geissler R, Barres BA, Wernig M (2013) Generation of oligodendroglial cells by direct lineage conversion. Nat Biotechnol 31:434-439. CrossRef Medline

Zhang SC, Ge B, Duncan ID (1999) Adult brain retains the potential to generate oligodendroglial progenitors with extensive myelination capacity. Proc Natl Acad Sci U S A 96:4089-4094. CrossRef Medline

Zhou L, Lovell KM, Frankowski KJ, Slauson SR, Phillips AM, Streicher JM, Stahl E, Schmid CL, Hodder P, Madoux F, Cameron MD, Prisinzano TE, Aubé J, Bohn LM (2013) Development of functionally selective, small molecule agonists at kappa opioid receptors. J Biol Chem 288:3670336716. CrossRef Medline 